\title{
Social and behavioral consequences of mask policies during the COVID-19 pandemic
}

Cornelia Betsch, ${ }^{a, b, *}$ Lars Korn, ${ }^{a, b}$ Philipp Sprengholz, ${ }^{a}$ Lisa Felgendreff, ${ }^{a, b}$ Sarah Eitze, ${ }^{a, b}$ Philipp Schmid, ${ }^{a, b}$ Robert Böhm ${ }^{c, d, e}$

${ }^{a}$ Media and Communication Science, University of Erfurt, 99089 Erfurt, Germany

${ }^{b}$ Center for Empirical Research in Economics and Behavioral Sciences, University of Erfurt, 99089 Erfurt, Germany

c Department of Psychology, University of Copenhagen, 1353 Copenhagen, Denmark

d Department of Economics, University of Copenhagen, 1353 Copenhagen, Denmark

e Copenhagen Center for Social Data Science (SODAS), University of Copenhagen, 1353

Copenhagen, Denmark

*Correspondence to: cornelia.betsch@uni-erfurt.de

\section{Classification}

Social Sciences: Psychological and Cognitive Sciences

\section{Keywords}

COVID-19, face masks, policy, pandemic

Please note: Submitted manuscript without peer-review. 


\begin{abstract}
Mandatory and voluntary mask policies may have yet unknown social and behavioral consequences related to the effectiveness of the measure, stigmatization, and perceived fairness. Serial cross-sectional data (04/14-05/26/20) from nearly 7,000 German participants demonstrate that implementing a mandatory policy increased actual compliance despite moderate acceptance; mask wearing correlated positively with other protective behaviors. A preregistered experiment $(n$ $=925$ ) further indicates that a voluntary policy would likely lead to insufficient compliance, would be perceived as less fair, and could intensify stigmatization. A mandatory policy appears to be an effective, fair, and socially responsible solution to curb transmissions of airborne viruses.
\end{abstract}

\title{
Introduction
}

Recommendations to wear non-medical face masks to control the spread of COVID-19 vary widely and have changed over time (1). At the beginning of the pandemic, some countries advised against wearing masks, partly because the supply of highly protective medical N95 masks was too low (1). In June 2020, World Health Organization has changed recommendations and now recommends healthy people to wear non-medical masks, e.g., in settings where physical distancing cannot be achieved (2). Some countries such as Germany require masks in supermarkets and public transport. Evidence has accumulated that strategies targeting the susceptible population can effectively contribute to the containment of the outbreak (3). As even non-medical masks reduce the spread of droplets and infectious aerosols $(4,5)$, mask wearing can protect others from contracting the virus (6) even though they do not prevent the maskwearing person from infection. As high compliance is needed to make it an effective measure (5), policies that encourage or enforce mask wearing need to be in place. Yet, little is known about the behavioral consequences of voluntary vs. mandatory mask policies and of the social evaluation processes that take place under either policy (7). Therefore, we report data assessed to support the German government and other regulatory bodies to gain insights into public opinion and acceptance of measures during the COVID-19 pandemic to facilitate evidence-based policy making and shaping communication efforts to justify policies (8).

\section{Results}

The reported test statistics are accompanied by effect sizes, i.e., Spearman correlation coefficient $\left(r_{\mathrm{s}}\right)$, odds ratio $(O R)$ for logistic regressions, $\eta_{\mathrm{p}}^{2}$ for analyses of variance (F-tests), Cohen's $d_{\mathrm{c}}$ for $t$ tests, $\beta$ for ordinary least-squares (OLS) regressions, and Cramer's $V\left(V_{c}\right)$ for $X^{2}$ tests; $p<0.05$ is considered statistically significant.

Figure 1 shows data from a weekly cross-sectional survey with $N=6,973$ German participants (approx. $n=1,000$ per week; online sample, quota-representative for age $\times$ gender and federal state in Germany, 04/14-05/26/20; for details see R markdown file) (9). At the onset of the COVID-19 outbreak, mask-wearing behavior was minimal (Fig. 1A). It increased steeply when a mandatory policy was enforced after April 27, 2020. Moreover, contrary to negative expectations (1), individuals wearing masks exhibited other protective behaviors more often (Fig. 1B). Given the moderate degree of acceptance (Fig. 1C) but apparent effectiveness of a mandatory policy, and the high public awareness of the social implications of mask wearing (Fig. 1D), it is necessary to explore the social consequences of mask policies, such as stigmatization (i.e., negative emotional responses, social labeling, or prejudicial attitudes (10)) toward people with and without masks (11), and perceived fairness.

Therefore, in an experiment included in the weekly survey on May 26/27, 2020, we focused on the following three questions: (i) Do policies affect stigmatization (i.e., do they intensify negative emotional responses, prejudicial attitudes, or social labeling toward people wearing or not wearing masks)? We assumed that mask wearing is a social contract and expected that people 
who fail to comply will be more likely to be stigmatized. We therefore assessed the warmth felt towards the other (11); how pro/-antisocial the other person was perceived as being (12); whether the other person was more likely to be regarded as sick or as a member of a risk group. (ii) As a second question, we inspected how the policies affect perceived fairness and whether the particularly vulnerable risk groups suffer under either policy (13). Finally, (iii) we investigated how policies affect compliance (i.e., the willingness to wear a mask given the specific policy). The experiment was pre-registered (https://aspredicted.org/blind.php?x=vx6rc4); we report tests of all pre-registered hypotheses and flag additional analyses as exploratory.

A total of $n=925$ participants faced a realistic scenario in which they had to imagine being in the fruit department of their local grocery store with one other person. We randomized whether there was a mandatory or voluntary mask policy in place, and whether or not the other person was wearing a face mask.

The results demonstrate that individuals with greater prosocial concerns (12) reported wearing masks in their everyday lives more frequently, $r_{\mathrm{s}}=0.15, p<0.001$. In the hypothetical scenario, however, prosociality did not predict future mask-wearing $(O R=1.01$, stand. err. $=0.01 p=$ 0.343 ) under either policy (interaction policy by prosociality: $O R=1.03$, stand. err. $=0.02, p=$ $0.074)$. However, participants perceived others with face masks as more prosocial than those without, irrespective of mask policy (Fig. 2A; main effect others' mask wearing behavior: $F(1,921)$ $=98.66, p<0.001, \eta_{\mathrm{p}}^{2}=0.097$; interaction policy by others' mask-wearing behavior: $F<1, n s$ ). Relatedly, participants who reported wearing a mask frequently in their everyday life perceived greater warmth toward others who also wear a mask than toward others who do not (Fig. 2B; interaction participant's mask-wearing behavior by others' mask-wearing behavior: $\beta=0.25, p<$ $0.001)$. This indicates that people who adhere to the social contract of wearing a mask tend to socially 'reward' each other but 'punish' others who do not wear a mask, irrespective of the mask policy in place. As more people reported being unwilling to wear a mask under a voluntary policy (77\% compliance compared to $96 \%$ under a mandatory policy, $X^{2}(1, n=925)=71.97, p<0.001$, $\left.V_{c}=0.279\right)$, more people may be subject to negative social evaluation under a voluntary policy (i.e., stigmatization could increase). In line with this, social labelling partially increased, as another person wearing a mask was more likely perceived as belonging to a risk group under a voluntary as opposed to a mandatory policy (Fig. 2C; interaction policy by others' mask-wearing behavior: $\left.F(1,921)=8.88, p=0.003, \eta_{\mathrm{p}}^{2}=0.010\right)$; however, they were not regarded as more likely to be infected with COVID-19 (Fig. 2D; $F<1, n s$ ).

Finally, participants perceived a mandatory mask policy as fairer than a voluntary mask policy (paired $t$-test; $t(923)=12.59, p<0.001, d_{c}=0.414$ ); explorative analyses suggested that this was especially pronounced for participants belonging to a risk group (Fig. 2E; interaction risk group by mask policy: $\left.F(1,922)=13.55, p<0.001, \eta_{\mathrm{p}}^{2}=0.014\right)$. Contrary to what was expected, the risk group's perceived susceptibility did not increase compared to a priori susceptibility, given either policy (interaction policy by risk group: $F(1,913)=2.87, p=0.091, \eta_{\mathrm{p}}^{2}=0.003$ ); instead, both groups felt more susceptible when the other person did not wear a mask (exploratory main effect others' mask-wearing behavior: $\left.F(1,913)=33.05, p<0.001, \eta_{\mathrm{p}}^{2}=0.035\right)$.

\section{Discussion}

In summary, the results from both data analyses indicate that, independent from policies, wearing masks is a social contract wherein compliant people perceive each other more positively, and non-compliance is socially sanctioned. Mask wearing is also related to adhering to other protective behaviors, and it signals prosocial concerns. This is consistent with previous work from the SARS-pandemic demonstrating that more empathic people tend to wear more masks (14) and that empathy can be regarded as a prerequisite for prosocial behavior (15). It needs to be noted that the results are based on self-reported survey data, not real-life observations. Thus, the answers may only approximate how people would actually feel or what they would actually do 
under different policies. Nevertheless, they provide a useful estimate of the policies' potential social and behavioral consequences. Modeling results suggest that "universal (80\%) adoption of moderately (50\%) effective masks could prevent on the order of $17-45 \%$ of projected deaths over two months" (calculated for New York state) (5). While uptake under a voluntary policy is reasonably high, it is still not sufficient to meet these required thresholds (5). Importantly, since mask wearing is a social contract (11), high uptake is necessary to prevent stigmatization. While this social dynamic can in fact increase mask wearing under a voluntary policy as well, it comes at the cost of social pressure, and it could increase the potential for polarization (e.g., when not wearing masks becomes a social sign of rejecting measures, see Fig. 1A).

In conclusion, should countries or communities want people to wear masks (e.g., to curb local outbreaks or to reduce transmission in future waves of the pandemic), introducing a mandatory policy along with explicit communication of the benefits of mask wearing (risk reduction, mutual protection, positive social signaling) and the benefits of the mandatory policy (fairness, less stigmatization, higher effectiveness) appears advisable.

\section{Materials and Methods}

Materials (including information about all measures and experimental manipulations), data, and the annotated data analysis code ( $R$ markdown), including code for all figures and corresponding analyses, are available at https://osf.io/dvbrn/. Data reported here are part of Germany's weekly COVID-19 Snapshot Monitoring (9).

\section{Acknowledgments}

Germany's COVID-19 Snapshot Monitoring (COSMO) is a joint project of the University of Erfurt (Cornelia Betsch (PI), Lars Korn, Philipp Sprengholz, Philipp Schmid, Lisa Felgendreff, Sarah Eitze), the Robert Koch Institute (Lothar H. Wieler, Patrick Schmich), the Federal Centre for Health Education (Heidrun Thaiss, Freia De Bock), the Leibniz Centre for Psychological Information and Documentation (Michael Bosnjak), Science Media Center (Volker Stollorz), the Bernhard Nocht Institute for Tropical Medicine (Michael Ramharter), and the Yale Institute for Global Health (Saad Omer). 


\section{References}

1. S. Feng, et al., Rational use of face masks in the COVID-19 pandemic. The Lancet Respiratory Medicine 8, 434-436 (2020).

2. W. H. Organization, "Advice on the use of masks in the context of COVID-19: interim guidance, 5 June 2020" (World Health Organization, 2020).

3. B. F. Maier, D. Brockmann, Effective containment explains subexponential growth in recent confirmed COVID-19 cases in China. Science 368, 742-746 (2020).

4. N. H. L. Leung, et al., Respiratory virus shedding in exhaled breath and efficacy of face masks. Nature Medicine 26 (2020).

5. S. E. Eikenberry, et al., To mask or not to mask: Modeling the potential for face mask use by the general public to curtail the COVID-19 pandemic. Infectious Disease Modelling 5, 293308 (2020).

6. K. A. Prather, C. C. Wang, R. T. Schooley, Reducing transmission of SARS-CoV-2. Science, eabc6197 (2020).

7. C. R. Sunstein, The meaning of masks. Journal of Behavioral Economics for Policy 4, 5-8 (2020).

8. C. Betsch, How behavioural science data helps mitigate the COVID-19 crisis. Nature Human Behaviour 4, 438-438 (2020).

9. C. Betsch, L. H. Wieler, K. Habersaat, Monitoring behavioural insights related to COVID-19. The Lancet (2020) https:/doi.org/10.1016/S0140-6736(20)30729-7 (April 5, 2020).

10. W. W. S. Mak, C. Y. M. Poon, L. Y. K. Pun, S. F. Cheung, Meta-analysis of stigma and mental health. Social Science \& Medicine 65, 245-261 (2007).

11. L. Korn, R. Böhm, N. W. Meier, C. Betsch, Vaccination as a social contract. Proceedings of the National Academy of Sciences of the United States of America (in press).

12. R. E. De Vries, The 24-item brief HEXACO inventory (BHI). Journal of Research in Personality 47, 871-880 (2013).

13. N. Daniels, Justice, Health, and Healthcare. The American Journal of Bioethics 1, 2-16 (2001).

14. D. Lee-Baggley, A. DeLongis, P. Voorhoeave, E. Greenglass, Coping with the threat of severe acute respiratory syndrome: Role of threat appraisals and coping responses in health behaviors: Coping with the threat of SARS. Asian Journal of Social Psychology 7, 9-23 (2004).

15. N. Eisenberg, P. A. Miller, The relation of empathy to prosocial and related behaviors. Psychological Bulletin 101, 91-119 (1987). 
A Wearing masks often or always

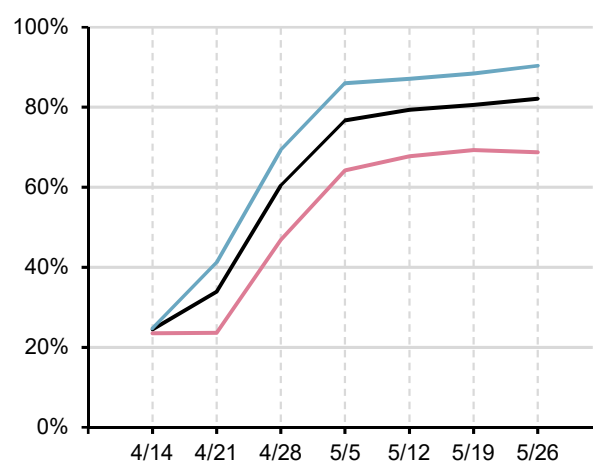

C Identifying masks as effective means of protection and supporting mandatory wearing

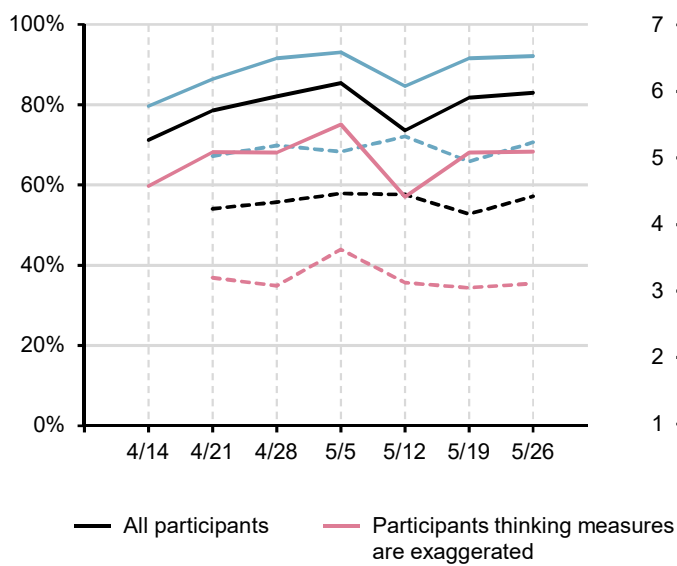

B Odds ratios for wearing masks relating to other protective behaviors

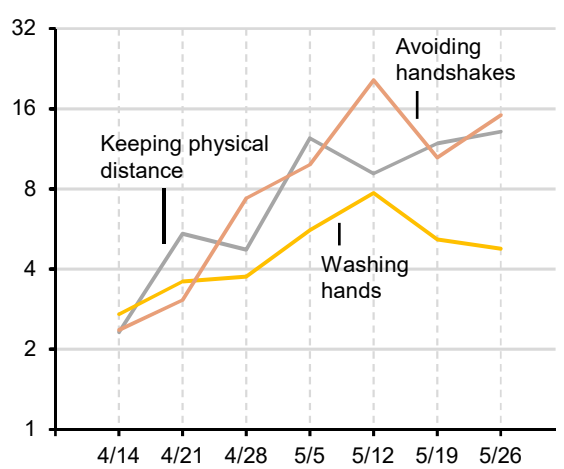

D Wearing masks to protect others and oneself

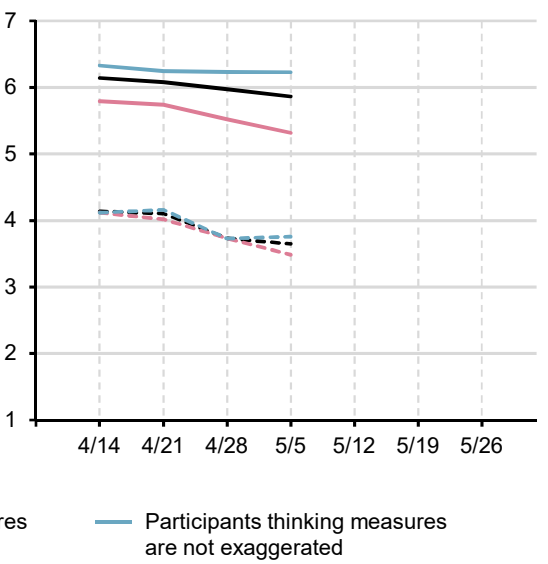

Figure 1. Behavior, knowledge and attitudes related to wearing masks in Germany during the COVID-19 pandemic.

Note: Data from Germany's COVID-19 Snapshot Monitoring (9) with $n_{04 / 14}=1032, n_{04 / 21}=1006$, $n_{04 / 28}=1018, n_{05 / 05}=1007, n_{05 / 12}=1013, n_{05 / 19}=972, n_{05 / 26}=925$. Masks wearing became mandatory in public transport and shops starting on 27th April and increased steeply (A). For people wearing masks, the probability of hand washing was between 2.71 and 7.73 times greater than for people not wearing masks; for handshakes, the probability was between 2.37 and 20.50 times greater; and for physical distancing, odds were between 2.32 and 13.13 times greater (B). Despite increasing compliance with mask-wearing requirements and widespread agreement that wearing masks is effective, support for mandatory policies remained at a moderate level throughout and largely differed between people who fully agreed with all measures and those who rejected them (C). Finally, knowledge that fabric masks do not protect the wearer but others became common among the public (D). Perceived exaggeration of policy measures based on median-split at each data collection (red vs. blue lines). Panel B displays odds ratios from binary logistic regressions, in which mask wearing predicted other behaviors. 
A
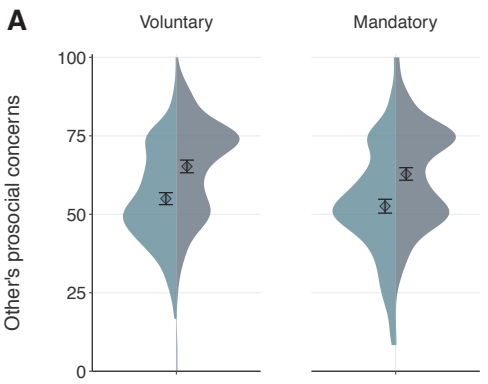

Other's mask
wearing behavior No mask $\square$ Mask
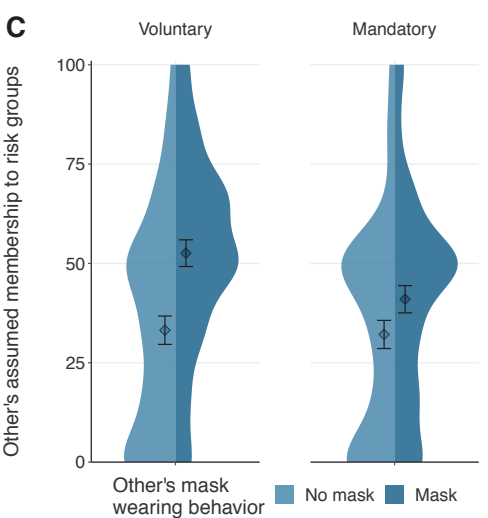

B

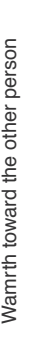

Voluntary

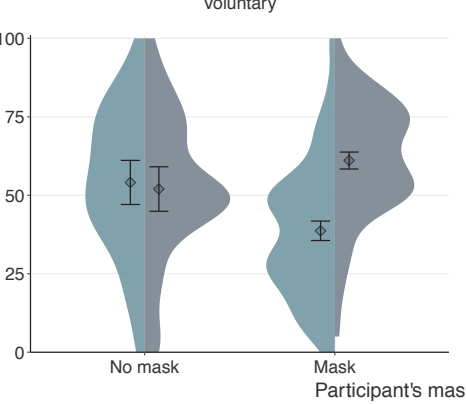
Other's mask
wearing behavior
No mask $\square$ Mask
Mandatory

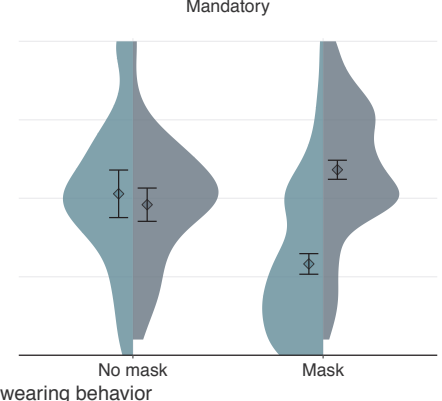

D

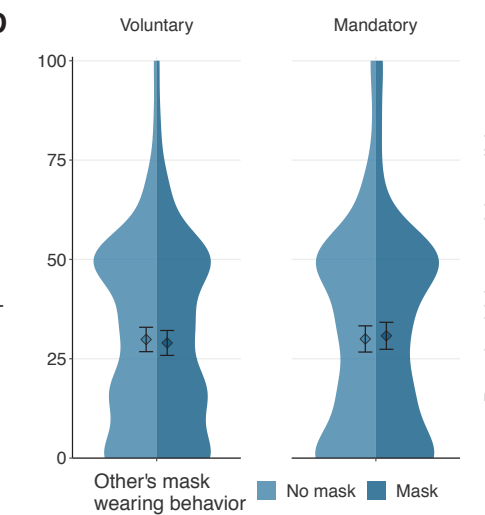

E

Voluntary

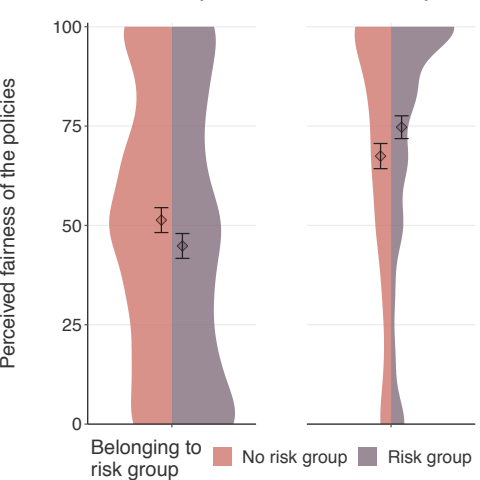

Figure 2. Social and behavioral consequences of mask wearing and mask-wearing policies Note: Participants imagined a grocery shopping situation wherein mask wearing was either mandatory or voluntary, and another person either did or did not wear a mask $\left(n_{05 / 26}=925(9)\right.$ ). Participants' frequency of mask wearing and membership in a risk group were also controlled for. Points represent mean values, error bars represent $95 \%$ confidence intervals. The colored areas represent rotated kernel density distributions of individual responses. Results illustrate that, independent from the policy, others wearing masks were perceived as more prosocial (A); mask wearing was perceived as a social contract, as those who complied with it socially "rewarded" each other but "punished" others who did not wear a mask (B). Only when mask wearing was voluntary did it partially affect stigmatization (others wearing a mask were judged as belonging to the risk group (C), but not as sick (D)). The voluntary mask policy was judged as less fair, especially by risk groups (E). All dependent variables were normalized to a range from $0-100$. 